\title{
Merit of PHY-MAC Cross-Layer Carrier Sensing: A MAC-Address-based Physical Carrier Sensing Scheme for Solving Hidden-Node and Exposed-Node Problems in Large-Scale Wi-Fi Networks*
}

\author{
An Chan and Soung Chang Liew \\ Department of Information Engineering, The Chinese University of Hong Kong \\ \{achan5,soung\}@ie.cuhk.edu.hk
}

\begin{abstract}
This paper examines how various carrier-sensing schemes affect the exposed-node (EN) and hidden-node (HN) phenomena. In the process, we identify a new carrier-sensing mechanism for alleviating EN and HN that is more effective than previously proposed schemes. This scheme, referred to as the MP scheme, uses MAC-address-based Physical carrier sensing to determine if the medium is busy. In MP, the addresses of transmitter and receiver of a packet are incorporated into the PHY header. Making use of this address information for its carrier-sensing operation, a node can drastically reduce the detrimental effects of EN and $H N$. In ns2 simulations, MP yields superior throughput and fairness performance that exceed our original expectation. Specifically, we find that the total network throughput achieved by MP is more than twice that of the $802.11 \mathrm{~b}$ basic-access mode, and more than four times that of the $802.11 b$ RTS/CTS mode, under various node-density and packet-size assumptions. At the same time, the Jain's Fairness Index of MP is almost twice that of the $802.11 \mathrm{~b}$ basic-access and RTS/CTS modes. We believe this is a first paper to propose and investigate the merit of cross-layer carrier sensing that jointly makes use of the attributes of the physical and MAC layers in its operation.
\end{abstract}

\section{Introduction}

Exposed-Node (EN) and Hidden-Node (HN) phenomena are known to affect the throughput and fairness performance of large-scale Wi-Fi networks in a fundamental manner [1] [2]. Sections 2.2 and 2.3 provide explanation on how EN and $\mathrm{HN}$ arise in a Wi-Fi network. A main contribution of this paper is the demonstration of the merit of "PHY-MAC cross-layer carrier sensing". In particular, we propose and investigate a novel scheme that exploits cross-layer carrier sensing to alleviate $\mathrm{EN}$ and $\mathrm{HN}$ effectively.

We refer to our proposal as the MP scheme to highlight its salient feature: MAC-address-based Physical carrier sensing. In traditional IEEE 802.11 physical carrier sensing, a node declares the medium to be busy if the detected power is above *This work was supported by the Areas of Excellence scheme (Project Number AoE/E-01/99) and the Competitive Earmarked Research Grant (Project Number 414305) established under the University Grant Committee of the Hong Kong Special Administrative Region, China. a threshold and/or the PHY header of a packet can be detected successfully [3]. In MP, a node also looks into the transmitter and receiver addresses of the transmission before declaring the medium to be busy. In particular, a node selectively ignores the detected transmission for certain transmitterreceiver pairs if it deems that there will be no collision between its transmission and the detected transmission. This significantly alleviates EN to the extent that the originally non-scalable 802.11 network becomes much more scalable with MP. That is, whereas the total network throughput of 802.11 quickly reaches a plateau as the number of wireless links in a given fixed area increases, the throughput of MP continues to increase proportionately.

A scheme previously considered by our research group, Selective Disregard of NAV (SDN) [1], is also scalable. SDN depends on RTS/CTS exchange to perform the MAC-addressbased carrier sensing to remove EN. RTS/CTS exchange incurs significant overhead for data transmission, especially when the data packet size is small. It is therefore not suitable for applications with small packets, such as Voice-over-IP (VoIP) [4]. In contrast, MP removes EN without using RTS/CTS. As an example, in our ns 2 simulations of a $5 \times 5$ Wi-Fi cellular network, given a packet loss-rate requirement of $1 \%, 40$ out of 50 VoIP sessions being simulated can meet the requirement with MP, but none can meet the requirement with SDN. We will show in this paper that MP is scalable for both large and small packets. In addition, we will also show that MP can achieve superior fairness performance when it is integrated with a Hidden-node Free Design (HFD) [5] to solve the HN problem.

The rest of this paper is organized as follows. Section 2 summarizes the salient features of MP and provides background on $\mathrm{EN}, \mathrm{HN}$, including how the carrier sensing operations in different schemes affect them. Section 3 describes the MP scheme in detail. Simulation results are presented in Section 4, with analytical discussions given in Section 5. Section 6 concludes this paper.

\section{Background and related work}

$\mathrm{HN}$ and $\mathrm{EN}$ in 802.11 networks have formally been defined using a graph model in [2]. Here, we only provide a general view for the relationship between carrier sensing and HN/EN.

Table I summarizes the operation of different carriersensing schemes. We briefly overview the content of the table 
Table I

Carrier sensing in different schemes

\begin{tabular}{|l|c|c|c|c|}
\hline \multicolumn{1}{|c|}{ Scheme } & PCS & VCS & $\begin{array}{c}\text { MAC-Address- } \\
\text { based CS }\end{array}$ & $\begin{array}{c}\text { HN } \\
\text { Free }\end{array}$ \\
\hline 802.11 Basic-Access Mode & $\sqrt{ }$ & & & \\
\hline 802.11 RTS/CTS Mode & $\sqrt{ }$ & $\sqrt{ }$ & & \\
\hline SDN & & $\sqrt{ }$ & $\sqrt{ }$ & \\
\hline SDN+HFD & RTS/CTS Only & $\sqrt{ }$ & $\sqrt{ }$ & $\sqrt{ }$ \\
\hline MP & $\sqrt{ }$ & & $\sqrt{ }$ & $\sqrt{ }$ \\
\hline
\end{tabular}

before providing more details in subsections 2.1, 2.2 and 2.3. The physical carrier sensing (PCS) in the 802.11 basic-access mode is such that a node will not initiate a transmission when it senses another packet with power exceeding a threshold and/or the PHY header can be decoded (note: the whole packet needs not be received correctly). In the 802.11 RTS/CTS mode, Virtual Carrier Sensing (VCS) is carried out by RTS/CTS exchange. A node looks into the Network Allocation Vector (NAV) field of a detected RTS/CTS frame and suspends its MAC countdown for a period corresponding to the NAV value [3]. In SDN, PCS is suspended and only VCS is used. However, SDN selectively disregards the NAVs of some detected RTS/CTS frames. In particular, if the node deems the link as indicated by the transmitter and receiver MAC addresses in the RTS/CTS frame to have no mutual interference with the link the node intends to transmit on, it will disregard the NAV. As indicated by Table I, SDN can be combined with a Hidden-node Free Design (HFD) to eliminate HN (i.e., the SDN+HFD entry) [2] [5]. Finally, the MP scheme considered in this paper is similar to the SDN+HFD scheme with the difference that PCS is used rather than VCS, so that the RTS/CTS overhead is eliminated.

\subsection{Summary of the distinguishing features of MP}

Despite the similarity between MP and SDN+HFD, MP has its own salient features that make it distinct from SDN+HFD. These features have significant implications for performance.

SDN+HFD is a combination of SDN and HFD. It uses RTS/CTS frames to perform MAC-address-based carrier sensing to remove EN. MP, on the other hand, does not use RTS/CTS exchange. MP exploits PHY-MAC cross-layer design (available in some commercial 802.11 chips - e.g., Atheros Chip) to perform MAC-address-based Physical carrier sensing. It alleviates $\mathrm{EN}$ and $\mathrm{HN}$ with less overhead.

In addition to large overhead, SDN also needs special control packets, called Power-Exchange (PE) packets, to collect the neighboring link-reception power information [1]. Essentially, each node constructs a power-transfer matrix for the nodes within its neighborhood, the $(i, j)$ component of which corresponds to the power transmitted by node $i$ that is received by node $j$. With the information in the power matrix, SDN can selectively ignore transmissions by other links that will not induce mutual collisions, even though the links could be heard. This is a fundamental key to enable scaled performance of the network [1][2]. One of the simplicities of MP as compared to SDN is that MP does not require special PE packets to derive the power-transfer matrix. Such information can be implicitly deduced from its PHY-MAC cross-layer carrier-sensing. The details of such implicit derivation will be described in a separate paper due to space limitation here.
As will be detailed later in Section 4, it turns out that besides its relative simplicity as compared with SDN, MP also has much better throughput and fairness performance than previously proposed schemes, including SDN and SDN+HFD For example, from our ns2 simulations, MP can achieve twice the throughput of SDN+HFD with better fairness performance. The system throughput of MP is comparable to that of SDN, but its Jain's Fairness Index is almost twice that of SDN.

\subsection{How carrier sensing affects $E N$}

In traditional 802.11 carrier sensing, the MAC addresses of the sensed packet do not matter. EN occurs when the carrier- sensing mechanism, which can be PCS or VCS, disallows simultaneous transmissions by non-interfering links [2]. An example of EN is shown in Fig. 1. IR is the interference range of a link. Any transmission by other links inside the IR of the link will interfere with the transmission of the link. Specifically, $I R=(1+\Delta) d[6]$ for a link with distance $d$, where $\Delta$ is a distance margin for interference-free reception. In this example, there are two wireless stations, $\mathrm{N} 1$ and $\mathrm{N} 2$, and two access points, AP1 and AP2. Links (N1, AP1) and (N2, AP2) are inside the carrier-sensing (CS) range but outside the IR of each other. So, simultaneous transmissions by the two links are not allowed, even though there is no mutual interference. We say that (N1, AP1) is exposed to (N2, AP2), and vice versa. EN is very common in the 802.11 basic-access mode as well as the RTS/CTS mode, and it is the fundamental factor limiting scalability of the Wi-Fi networks [1] [2] due to inefficiency in spatial reuse.

SDN solves the EN problem. It suspends PCS and uses only VCS. Upon reception of a VCS (RTS/CTS frame), instead of simply declaring the medium to be busy and respecting the NAV blindly, a node will look at the MAC addresses of the RTS/CTS frame to see if the transmitting link has an interfering relationship with the link the node intends to transmit on. If not, it will simply disregard the RTS/CTS frame. Consider the example in Fig. 1. Suppose that N1 is transmitting a packet to AP1, and N2 hears the CTS from AP1 to N1. In SDN, since the two links have no mutual interference, N2 will not set NAV and will go ahead to send a packet to AP2. The reader is referred to [1] for the detailed operation of SDN.

MP has no RTS/CTS exchange. As in SDN, a node also looks at the MAC addresses of the received packet and then selectively respects the PCS (not VCS) only if there is an interference relationship between the transmitting link and the link the node intends to transmit on. Therefore, MP also eliminates EN as SDN does, with the advantage that there is no RTS/CTS overhead.

\subsection{How carrier sensing affects $H N$}

In 802.11 networks, $\mathrm{HN}$ occurs when the CS mechanism fails to prevent simultaneous transmissions of interfering links. Fig. 2 gives an example. Consider the two links (N1, AP1) and (N2, AP2). The dashed-line circle represents the CS range of N1. During the transmission of N1 to AP1, N2 could still send packets to AP2, since N2 is not within the CS range of N1. But AP1 and AP2 are inside the IR of each other. The 


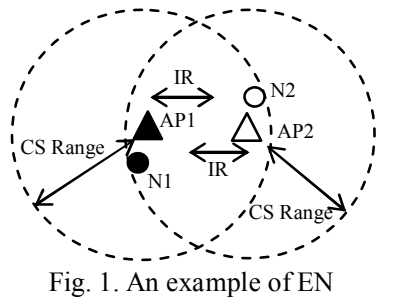

acknowledgment (ACK) from AP1 to N1 could collide with DATA from N2 to AP2 at AP2. We say that (N1, AP1) is hidden from (N2, AP2). As with EN, $\mathrm{HN}$ is also common in the conventional 802.11 network. The failure of carrier sensing to prevent interfering transmissions may cause unfair throughput performance among links and even bandwidth starvation [2] [7].

SDN alone does not eliminate the HN problem. In fact, it is particularly susceptible to HN during the RTS/CTS exchange since PCS is suspended.

HN can be eliminated by a Hidden-node Free Design (HFD) [5] which consists of two requirements. The first requirement is that the CS Range must be sufficiently large:

$$
\text { CSRange } \geq(3+\Delta) d_{\max }
$$

where $d_{\max }$ is the maximum link length in the network. This requirement is illustrated in Fig. 3. To prevent collisions, N1 and N2 must be able to sense each other. Therefore, the CS range should be at least $\left(2 d_{\max }+I R\right)$. Since $I R=(1+\Delta) d_{\max }$, inequality (1) follows.

Increasing the $\mathrm{CS}$ range alone cannot eliminate $\mathrm{HN}$ completely [5], since no matter how large the CS range is, it is still possible that a receiver is inside the CS range of a transmitting link, but its sender is not. To see this, in Fig. 3, shift AP2 and N2 to the right so that AP2 lies within the CS Range but not N2. In this case, the sender will send DATA to the receiver, but the receiver will not return an ACK, causing the sender to interpret the event as a collision [5]. To eliminate $\mathrm{HN}$ in this scenario, the second requirement is to enable the so-called Receiver Restart (RS) Mode, which is incorporated into some commercial 802.11 chip (e.g., Atheros Chip) but is usually not enabled by default.

With RS, the receiver switches to receive a new signal in the midst of receiving an old signal if the power of the new signal is sufficiently larger (say, $10 \mathrm{~dB}$ stronger) than that of the old one. Therefore, in the above example, provided the signal from $\mathrm{N} 2$ is sufficiently larger than the signal from AP1, AP2 will switch to receive the DATA from N2 and return an ACK. A reason why the RS Mode is by default usually not enabled in commercial products is as follows. Consider the example in Fig. 2 where we increase the CS range of $\mathrm{N} 1$ to cover AP2 but not large enough to cover N2. Suppose that N1 sends a packet to AP1 followed by a transmission of a small packet from N2 to AP2. As AP2 is covered by the CS range, $\mathrm{AP} 2$ would not respond to the packets sent from N2 with an ACK. Thus, the transmission in link (N1, AP1) would not be affected. But if RS Mode is enabled and the signal power of packet from N2 is $10 \mathrm{~dB}$ larger than that from N1 at AP2, then AP2 will receive the packet from N2 and respond with an ACK packet. The ACK packet causes collision at AP1 as AP1 is still receiving the packet from N1. In this case, instead of eliminating $\mathrm{HN}$, the network performance is further degraded.
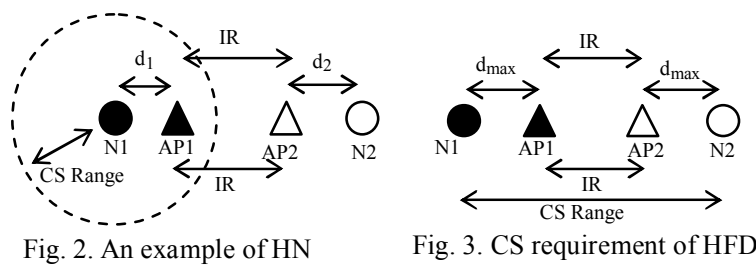

Fig. 3. CS requirement of HFD

When the CS range is sufficiently large (large enough to cover $\mathrm{N} 2$ in this example), however, this situation does not occur. HFD [5] basically specifies the CS range which when combined with the RS Mode will ensure $\mathrm{HN}$-free operation.

As indicated by Table I, HFD can be combined with SDN for the SDN+HFD scheme [2] to solve $\mathrm{EN}$ and $\mathrm{HN}$ simultaneously. In SDN+HFD, $\mathrm{HN}$ is removed and $\mathrm{EN}$ is alleviated but not eliminated entirely (note: the original SDN removes EN completely). The reader is referred to [2] for details of SDN+HFD.

MP can also be made HN-free by incorporating HFD. As indicated in Table I, this paper primarily investigates MP with HFD although MP without HFD is in principle also possible. That is, by MP, we mean MP+HFD in this paper.

\section{Description of MP protocol}

Before describing MP in detail, we first introduce a receiver's mechanism fundamental to the operation of MP, referred to as the Cross PHY-MAC Mode.

\subsection{Cross PHY-MAC mode}

In the conventional 802.11 schemes which implement strict layering, once a node detects a packet, the physical layer of the node will attempt to receive the whole packet before passing it to the MAC layer. The physical layer will not look at the MAC addresses which belong to the MAC header. The MAC layer will further pass the packet to the upper layer only if the MAC receiver address of the packet matches the node's MAC address.

Fig. 4 shows the address fields, which are inside the MAC header, in a DATA frame. Some commercial 802.11 chips (e.g., Atheros Chip) implement a "Cross PHY-MAC Mode" in the receiver design. With this mode, the physical layer may actually look at the MAC addresses to decide how to do carrier sensing. For example, it may choose to ignore packets from certain transmitter or packets to certain receiver when it decides whether the medium is busy or not. We refer to this as MAC- address-based Physical carrier sensing.

\subsection{MP protocol}

MP implements a form of MAC-address-based Physical carrier sensing which depends on both the transmitter and receiver addresses of the packet being sensed, as well the transmitter and receiver addresses of the packet the node intends to transmit. The dependency is similar to that in SDN [1] except that PCS is used in MP so that the RTS/CTS overhead in SDN can be eliminated. However, MP is not just a straight-forward, direct extension of SDN. Additional techniques are needed to enable MP, as described below.

For MAC-addressed-based PCS in MP, a node should be able to decode the MAC addresses once it decodes the PHY 
header. But in the conventional 802.11 (take IEEE $802.11 \mathrm{~b}$ as an example) basic-access mode, the data rate of the PHY header is $1 \mathrm{Mbps}$, with transmission range of $550 \mathrm{~m}$ (which corresponds to the carrier-sensing range), while the data rate of the MAC header, which is encapsulated inside data payload (see Fig. 4), is $11 \mathrm{Mbps}$, with transmission range of $232 \mathrm{~m}$. If the node is within the transmission range of the PHY header but outside the transmission range of the MAC header, the MAC addresses may not be decodable although the PHY header is decodable. Then, the node cannot perform PCS according to the MAC addresses. Therefore, for MP, the transmission range of $\mathrm{PHY}$ header and that of MAC addresses should be same. This means that the data rate of MAC addresses should be decreased to that of the PHY header. For simplicity, we propose to move the transmitter and receiver address fields to the PHY header so that they can be sent at $1 \mathrm{Mbps}$ as well (as shown in Fig. 5). Although the overhead of MP is larger than that of 802.11 basic-access mode after moving the MAC addresses to the PHY header, MP can still have rather significant throughput-fairness performance improvements over the 802.11 basic-access mode, as validated by simulation results presented in Section 4.

Fig. 6 shows a flowchart outlining the operation of MP. When the received signal power, $P_{p k t}$ exceeds a receiver detection threshold $P_{\text {thresh }}$, the node will attempt to receive the packet. The receiver detection threshold $P_{\text {thresh }}$ here serves the same function as Clear-Channel-Assignment (CCA) threshold in the 802.11 standard [8]. The difference is that exceeding $P_{\text {thresh }}$ here means there is possibly a "relevant" packet rather than a declaration that the channel is busy. The condition $P_{p k t}$ $>P_{\text {thresh }}$ triggers the receiver to try to detect the packet, and to decide whether the channel is busy by following the steps as shown in Fig. 6 and as detailed below:

(i) First, the receiver sees if the PHY header can be received correctly (using the HEC for parity check). If no, the node will treat the medium as busy and continue to monitor the packet until $P_{p k t}<P_{\text {thresh }}$. The fact that the PHY header cannot be received means there is perhaps a non-802.11 source (e.g., Microwave oven, Bluetooth) transmitting at the same frequency band) and they may cause collisions with the 802.11 transmissions. When $P_{p k t}<P_{\text {thresh }}$, following an EIFS (DIFS + ACK time), the node will go back to monitor the medium for another packet. In the state of continuous monitoring of the packet, the transmission mechanism and backoff counter are frozen until $P_{p k t}<P_{\text {thresh. In Fig. 6, we }}$ include an asterisk for states in which the transmission and backoff mechanism is frozen.

(ii) If the PHY header can be decoded correctly, the node

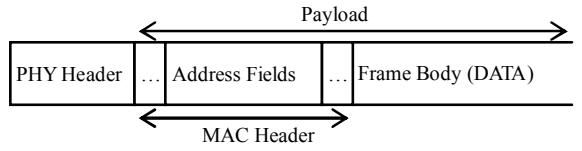

Fig. 4. Address fields in a DATA frame

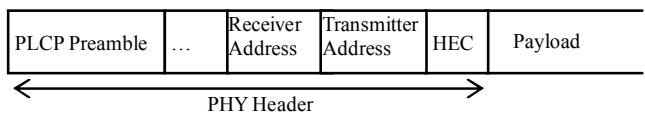

Fig. 5. PHY header of a DATA frame after adding MAC addresses next decides whether the packet is destined for it. If yes, the whole packet is received and passed to the upper layer. While the packet is being received, the channel is also declared to be busy so that the transmission and backoff mechanism is frozen.

(iii) If the packet is targeted for other nodes, the node decides whether this packet is from a link that has an interfering relationship with the link the node intends to transmit on. (a) If there is interference relationship, then the normal PCS operation as in the conventional 802.11 basic mode will be conducted, including setting the NAV according to the packet length information extracted from the PHY header. (b) If there is no interference relationship, the node will treat the medium as idle and go back to monitor the medium for the next packet. Thus, there is no asterisk for case (b), and the transmission and backoff countdown mechanism can still be continued because the medium is treated as idle.

For normal CCA in the standard 802.11, (i) and (ii) are same. For (iii), normal CCA will always declare the channel to be busy without checking if the packet is from a link with an interfering relationship with the node. However, MP will divide (iii) further into cases (a) and (b). In (b), the channel will not be declared to be busy. In addition, the receive detection threshold $P_{\text {thresh }}$ will be increased by an amount corresponding to the power of the current signal being heard this is part of the operation of the RS Mode: the new signal must be, say $10 \mathrm{~dB}$, above the current signal for the receiver to start receiving the new signal. When the current signal is no longer detected, $P_{\text {thresh }}$ is decreased back to the original value.

Note that the carrier sensing in MP requires the knowledge of the presence or absence of interference relationships among neighboring nodes. As mentioned in the previous section, this neighborhood links information can be discovered either by MAC-address-based PCS in MP or a distributed PowerExchange (PE) algorithm as described in [1]. With such information, a node can identify all neighborhood links with an interference relationship with it. Due to the limit of space,

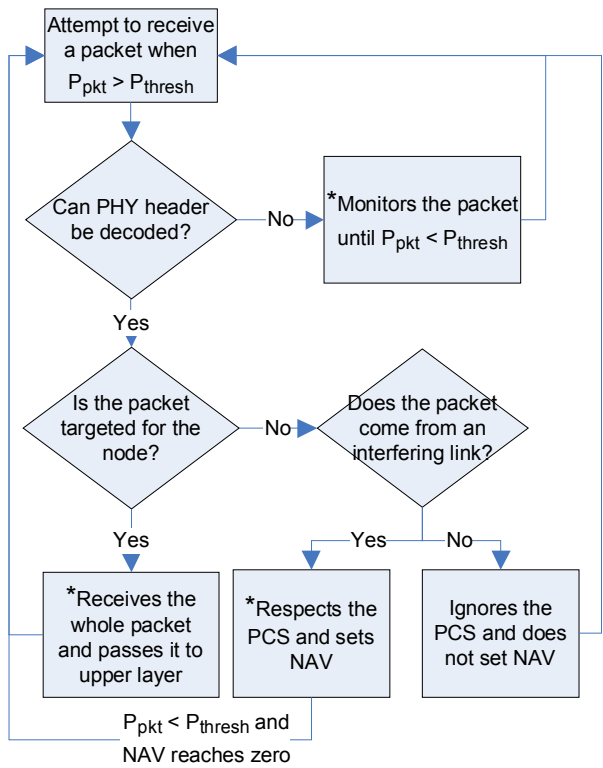

Fig. 6. Flowchart of the operation of MP 
the details will be deferred and described in another paper.

To make the wireless network HN-free in MP, the PCS range is set according to (1), and the RS Mode is enabled. As a result, HN during the reception of PHY header is removed. The large CS Range in (1), however, may cause excessive EN if it applies for the whole DATA frame. Therefore, after the PHY header is decoded, the MAC-address-based PCS in MP and the selective NAV setting kick in to alleviate EN.

\section{Simulations and discussion}

To evaluate the performance of MP, we have carried out simulations on ns2 [9]. To simplify our simulations, we model MP by integrating MAC and PHY headers, and send them together with the data rate of $1 \mathrm{Mbps}$. If we merely move the transmitter and receiver addresses from the MAC header to the PHY header as described in the exact MP protocol, the simulation performance of MP would be better as less overhead would be incurred. In our simulations, the data rates of PHY header and DATA payload are $1 \mathrm{Mbps}$ and $11 \mathrm{Mbps}$ respectively.

\subsection{Performance of MP and 802.11 basic-access mode}

In MP, MAC addresses are moved from MAC header to PHY header and are sent with $1 \mathrm{Mbps}$, and the overhead for data transmission becomes larger, especially when small data packets are sent. However, the net effect is that MP still yields significant performance improvement over the 802.11 basicaccess mode. In the following ns 2 simulations, we model VoIP over WLANs.

We set up a grid topology with $5 \times 5$ equal-sized cells inside a square area of $700 \mathrm{~m} \times 700 \mathrm{~m}$. An access point (AP) is placed at the center of each cell, with 50 wireless client stations are randomly placed in the $700 \mathrm{~m} \times 700 \mathrm{~m}$ square area with uniform distribution. Each client associates with the nearest AP. Thus, two clients are associated with an AP on average, and there are 50 bidirectional links (50 VoIP sessions) built up in total. VoIP of codec GSM 6.10 is modeled: for each session, 33-byte voice packets are generated with the rate of 50 packets per second [4]. Another 40 bytes IP/UDP/RTP header is added onto each voice packet before sending [4], so that the effective throughput of each VoIP link (either uplink, from client to AP, or downlink, from AP to client) in the absence of packet loss is $29.2 \mathrm{Kbps}$. For discussion purposes, we assume the loss rate of a session is to be less than $1 \%$. The number of VoIP sessions that meet this requirement will be examined.

Fig. 7 and 8 show the throughput of each link in $802.11 \mathrm{~b}$ basic-access mode and MP respectively. Table II shows that MP can support 15 more VoIP sessions than $802.11 \mathrm{~b}$ basicaccess mode. From this result, we can conclude that although MP has larger "header" overhead than 802.11 basic-access mode, MP still has better performance even when small data packets are sent. Table II also shows the numbers of VoIP sessions supported by other carrier sensing schemes. As predicted, 802.11b RTS/CTS, SDN, and SDN+HFD schemes can only support few or none of the VoIP sessions due to the large RTS/CTS overhead.

\subsection{Performance scalability}

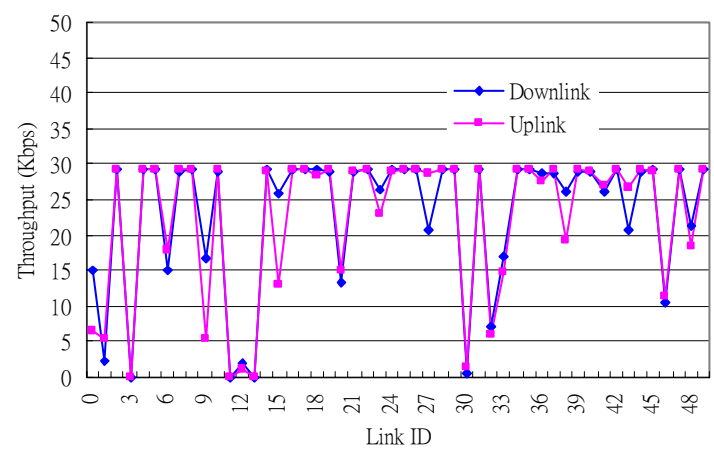

Fig. 7. Throughput of each bidirectional link in $802.11 \mathrm{~b}$ basic-access mode

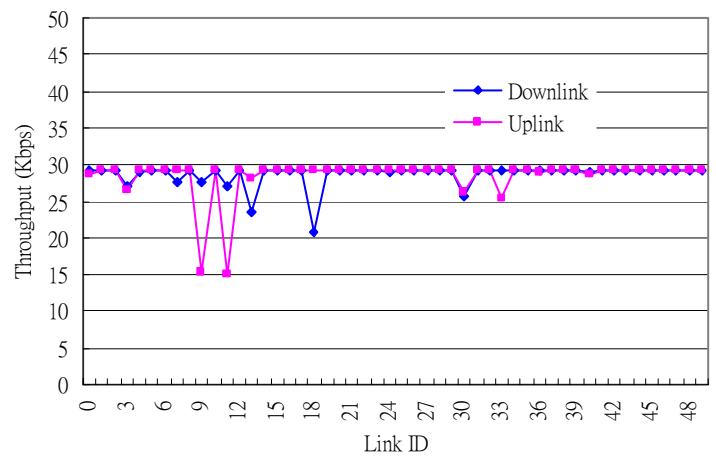

Fig. 8. Throughput of each bidirectional link in MP

Table II

Number of VOIP sessions with loss rate smaller than $1 \%$

\begin{tabular}{|l|c|}
\hline \multicolumn{1}{|c|}{ Scheme } & $\begin{array}{c}\text { No. of VoIP Sessions with less } \\
\text { than 1\% Packet Loss }\end{array}$ \\
\hline $802.11 \mathrm{~b}$ Basic-Access & 25 \\
\hline MP & 40 \\
\hline $802.11 b$ RTS/CTS & 0 \\
\hline$S D N$ & 0 \\
\hline$S D N+H F D$ & 3 \\
\hline
\end{tabular}

In this subsection, we consider the scalability of different schemes. Consider a square area of $\mathrm{D}$ by $\mathrm{D}$, with $\mathrm{M}^{2}$ equal-sized cells inside the area. Within the area, a total of $4 \mathrm{M}^{2}$ wireless client stations are randomly placed with a uniform distribution. An AP is located at the center of each cell. Each client station associates with the nearest AP. On average, four clients associate with each AP. We then generate a saturated UDP flow from each client to its associated AP.

In the simulation, $\mathrm{D}$ is set to be $700 \mathrm{~m}$ and $\mathrm{M}$ is set to be 3 , 4 and 5 progressively. We set the PCS range to be $640 \mathrm{~m}$ and VCS range to be $480 \mathrm{~m}$ which are the minimum ranges to satisfy the HFD when M is 3 [2]. The total throughput of the network is measured for different $M$ values. The UDP packets are of size 1460 bytes.

Fig. 9 shows the simulation results of the total network throughput with $95 \%$ confidence intervals. The total network throughput is defined as the total bytes, excluding PHY and MAC headers, received by all the receivers (i.e. the total $\mathrm{M}^{2}$ APs) divided by the simulation run time. As the value of $M$ increases (i.e., grid size decreases and number of cells increases), the total network throughput of $802.11 \mathrm{~b}$ RTS/CTS, basic-access and SDN+HFD schemes decreases slightly, showing that these schemes are not scalable. This 


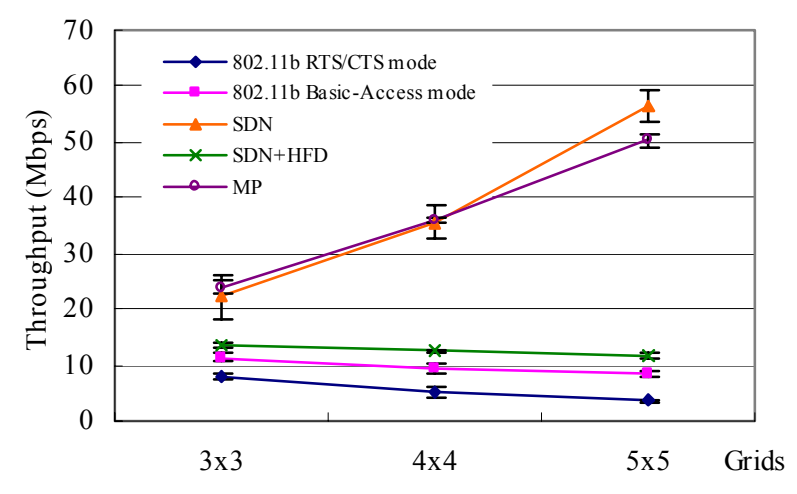

Fig. 9. Throughput of different schemes as value of $\mathrm{M}$ increases

phenomenon can be explained as follows. As the density of wireless nodes (including APs and client stations) increases, the average link distance between the client and its associated AP is shortened. The fixed PCS range and VCS range, however, cause unnecessary EN situations. In particular, the PCS and VCS start to cover more cells (as shown in Fig. 10) so that the nodes in adjacent cells are increasingly exposed to each other. The total network throughput cannot increase even though the number of cells increases.

As shown in Fig. 9 also, the total network throughput of SDN and MP increases as M increases. MP does not use RTS/CTS handshake, so the VCS range is not applicable to it. Furthermore, it can selectively respect the PCS of the DATA packet after the address fields are decoded, so the fixed PCS range does not cause excessive EN - that is, for two links that do not mutually interfere, even if they are within the PCS range of each other, they can still transmit simultaneously. SDN ignores the PCS of all packets. As with MP, the fixed VCS range does not cause EN. The SDN is indeed the most scalable scheme among the five shown in Fig. 9, as can be seen from the fact that the slope of the throughput graph of $\mathrm{SDN}$ is slightly greater than that of MP.

Fig. 11 shows the total network throughput of different schemes after applying Uniform Power Control (UPC). With UPC, we scale the PCS range and VCS range to exactly meet the required range of HFD when different values of $M$ are used. That means that as M increases, the PCS range and VCS range would decrease correspondingly to reduce EN. From the graph, we find that all five schemes are scalable after UPC is applied. MP now is as scalable as SDN. Recall that the MP studied here incorporates HFD. So, it alleviates EN and eliminates HN. The pure SDN, on the other hand, does not incorporate HFD and therefore incurs substantial HN. The throughput of MP is slightly larger than that of SDN.

We also use the Jain's Fairness Index [10] to measure the throughput fairness of the UDP flows in different schemes after UPC is applied. The Jain's Fairness Index is given in (2), where $n$ is the total number of links; $x_{i}$ is the normalized

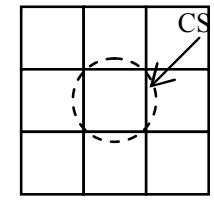

$3 \times 3$ Grid

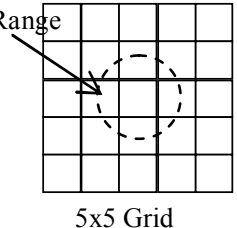

$5 \times 5$ Grid
Fig. 10. Same CS range covers more cells as value of $\mathrm{M}$

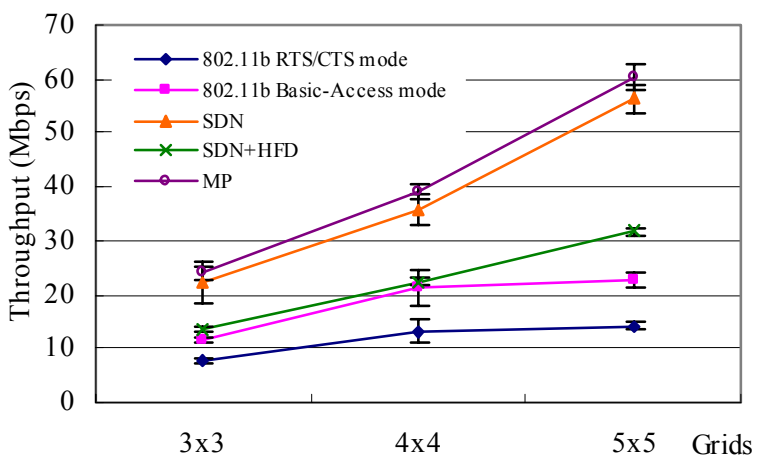

Fig. 11. Throughput of different schemes as value of M increases after Uniform Power Control

throughput for link $i, x_{i}=T_{i} / O_{i} ; T_{i}$ is the throughput of link $i$; and $O_{i}$ is the fairness throughput of link $i$ [10]. In our measurement, we define $O_{i}$ to be the same for all $i-$ i.e., it is the total network throughput divided by total number of links.

$$
\text { Jain's_Fairness__ndex }=\frac{\left(\sum x_{i}\right)^{2}}{n \sum x_{i}{ }^{2}}
$$

Fig. 12 shows the average fairness index of different schemes measured from the simulation results when $M$ is 5 . The $95 \%$ confidence intervals are represented by the line segments. From the chart, we find that MP and SDN+HFD have better fairness than other schemes, since HNs have been removed. The fairness of MP is better than that of SDN+HFD by around $10 \%$.

\subsection{Different DATA packet sizes measurement}

We have also carried out simulations for different UDP packet sizes: 50-byte, 210-byte, 500-byte and 1460-byte packets. We use 50-byte packets to simulate VoIP [4]. The topology used is the $5 \times 5$ grid. We fix the PCS range and VCS range at $550 \mathrm{~m}$ and $437 \mathrm{~m}$, respectively (i.e., without UPC).

In Fig. 13, the total network throughput of different schemes is shown with $95 \%$ confidence interval. When small UDP packets are used (the exact throughput of different schemes is shown in Table III), doing without RTS/CTS becomes more worthwhile. For example, MP has larger throughput than SDN (by 23\% for 50-byte packets), and more than twice the throughput of SDN+HFD (by $250 \%$ for 50 -byte packets).

\section{Further discussion of performance results}

In this section, we analyze the overhead saving of MP as compared to SDN+HFD. The essential difference between MP and SDN+HFD is that MP operates without RTS/CTS handshake before DATA packet transmission. Our analysis below predicts the throughput improvement in the single-cell situation (when there is only one AP) with reasonable accuracy. However, the drastic throughput advantage of MP over SDN+HFD in the multi-cell case, as observed in the simulation results in Section 4, cannot be explained by overhead saving alone.

We define overhead $(\mathrm{OH})$ as (3), where $T_{\text {trans }}$ is the total airtime needed to complete a transmission, and $T_{\text {data }}$ is the airtime used for transmitting the pure data content in DATA packet (i.e., packet transmission time minus the transmission 


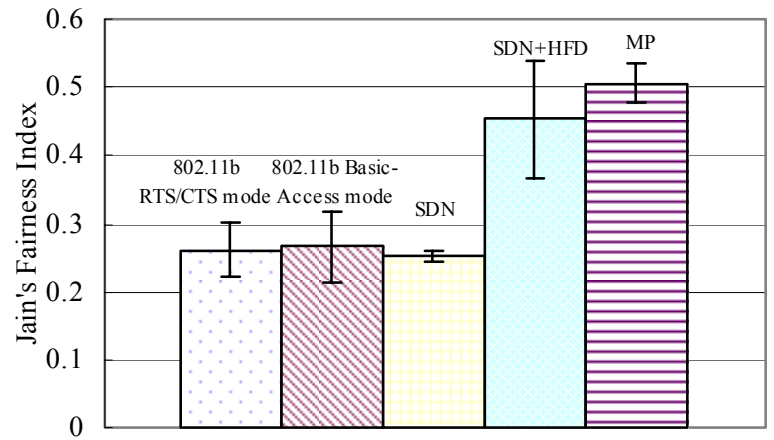

Fig. 12. Jain's Fairness Index of different schemes in $5 x 5$ topology

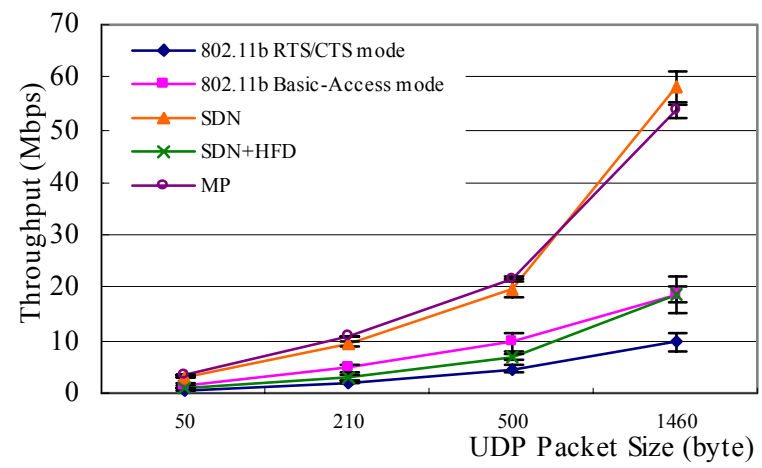

Fig. 13. Throughput of different schemes in $5 \times 5$ topology when different sizes of UDP packets are used

Table III

Average total network throughput of different schemes as small UDP packet used

\begin{tabular}{|l|c|c|c|}
\hline UDP Packet Size (Byte) & 50 & 210 & 500 \\
\hline 802.11 RTS/CTS (Mbps) & 0.68 & 2.20 & 4.68 \\
\hline 802.11 Basic Access (Mbps) & 1.61 & 4.83 & 9.69 \\
\hline SDN (Mbps) & 2.91 & 9.35 & 19.67 \\
\hline SDN+HFD (Mbps) & 1.02 & 3.05 & 6.99 \\
\hline MP (Mbps) & 3.58 & 10.95 & 21.82 \\
\hline
\end{tabular}

time for PHY, MAC headers and a CRC checksum which is appended to the packet). While $T_{\text {data }}$ are the same, $T_{\text {trans }}$ in MP and SDN+HFD are different. $T_{\text {trans }}$ in MP and SDN+HFD are given by (4) and (5), respectively.

$$
\begin{gathered}
O H=\left(\frac{T_{\text {trans }}}{T_{\text {data }}}-1\right) \times 100 \% \\
T_{\text {trans }}=T_{\text {backoff }}+T_{\text {DIFS }}+T_{\text {SIFS }}+T_{P A C K E T}+T_{A C K} \\
T_{\text {trans }}=T_{\text {backoff }}+T_{\text {DIFS }}+3 T_{\text {SIFS }}+T_{R T S}+T_{C T S}+T_{P A C K E T}+T_{A C K}
\end{gathered}
$$

where $T_{\text {backoff }}$ is the countdown time of the backoff counter before transmission starts; $T_{D I F S}$ is the time for Distributed Coordination Function (DCF) interframe space [4]; $T_{\text {SIFS }}$ is the time for short interframe space (SIFS); $T_{R T S}$ is the time for transmitting a RTS frame; $T_{C T S}$ is the time for transmitting a CTS frame; $T_{P A C K E T}$ is the time for transmitting a DATA packet ( $T_{\text {data }}$ plus the airtimes for PHY, MAC headers and the CRC checksum); and $T_{A C K}$ is the time for transmitting an ACK frame.

Note that $T_{P A C K E T}$ in MP is longer than in SDN+HFD. In MP, the MAC-address fields are in the PHY header which is sent with $1 \mathrm{Mbps}$, while in SDN+HFD, the MAC-address fields are in data payload which is sent with $11 \mathrm{Mbps}$.
We first look at the relationship between the overhead and the total network throughput in the single-cell case. In this case, all the nodes can hear each other's transmission, and there is no EN and HN. Under saturated traffic condition, the total throughput of MP and SDN+HFD and their overheads are related as follows:

$$
\rho_{M P}\left(1+O H_{M P}\right)=\rho_{S D N+H F D}\left(1+O H_{S D N+H F D}\right)
$$

where $\rho_{\mathrm{MP}}$ and $\rho_{\mathrm{SDN}+\mathrm{HFD}}$ are the total throughput of MP and SDN+HFD, and $O H_{M P}$ and $O H_{S D N+H F D}$ are the overheads of MP and SDN+HFD, respectively. The throughput improvement of MP over SDN+HFD is given by

$$
\frac{\rho_{M P}-\rho_{S D N+H F D}}{\rho_{S D N+H F D}}=\frac{O H_{S D N+H F D}-O H_{M P}}{1+O H_{M P}}
$$

Table IV shows the throughput improvements for various packet-payload sizes. The 1460-byte payload is the usual size for TCP packets. The 50-byte payload is typical for VoIP packets (including the voice payload and IP/UDP/RTP header) over 802.11 Wireless LAN (WLANs) [4]. The 210-byte payload is also used in some commercial VoIP products [11].

To validate the above analysis, we have performed ns2 simulations for MP and SDN+HFD schemes in a single-cell topology. The simulation results are given in Table V. We find that the results of the overhead analysis in Table IV match the results in Table $\mathrm{V}$ reasonably well. From the results, we confirm that MP can attain larger total throughput than SDN+ HFD, especially when the payload of DATA packet is small.

The single-cell overhead analysis presented above is a simple one which assumes packet collision probability is zero. Using similar techniques as in [12], we have performed analysis that takes into account the finite packet-collision probability. To conserve space, we only present the results of the analysis here. We calculate the normalized throughput of MP and SDN+HFD and compare them with the normalized throughput measured from ns2 simulation. The normalized throughput is defined as the calculated or simulated throughput divided by the "ideal" maximum throughput. The ideal maximum throughput is defined to be the throughput attainable if the airtime is fully occupied by successful data payload without collisions. In this single-cell scenario, the ideal maximum throughput is equal to the data rate of data payload, which is $11 \mathrm{Mbps}$ in our case. The results are shown in Fig. 14. From Fig. 14, we find that after taking the packet collision into account, the throughput of MP and SDN+HFD

Table IV

Overheads of MP and SDN+HFD when DATA frames with different sizes of packet-payload are transmitted

\begin{tabular}{|c|c|c|c|c|}
\hline Packet-Payload Size (Byte) & 50 & 210 & 500 & 1460 \\
\hline OH of MP (\%) & 2190 & 522 & 219 & 75 \\
\hline OH of SDN+HFD (\%) & 3260 & 776 & 326 & 112 \\
\hline $\begin{array}{c}\text { Throughput Improvement of MP } \\
\text { Contributed by OH Reduction (\%) }\end{array}$ & 46.7 & 40.8 & 33.5 & 21.1 \\
\hline
\end{tabular}

Table V

Average total network throughput of MP and SDN+HFD in single-cell simulation as different sizes of UDP packet are used

\begin{tabular}{|c|c|c|c|c|}
\hline UDP Packet Size (Byte) & 50 & 210 & 500 & 1460 \\
\hline MP (Mbps) & 0.571 & 1.68 & 3.18 & 5.87 \\
\hline SDN+HFD (Mbps) & 0.415 & 1.23 & 2.43 & 4.79 \\
\hline Throughput Improvement of MP (\%) & 37.6 & 36.6 & 30.9 & 22.5 \\
\hline
\end{tabular}


can be predicted analytically with much higher accuracy than the simple collision-free analysis we used above. This single-cell overhead analysis further validates the accuracy of our implementations of SDN+HFD and MP on ns2.

In the multi-cell case as studied in Section 4, interference and carrier sensing among the nodes in different cells may cause $\mathrm{HN}$ and EN - after all, dealing with these EN and $\mathrm{HN}$ are the main essence of MP and SDN+HFD. The comparison between MP and SDN+HFD is more complicated than can be captured by the overhead analysis alone. For example, in large packet size of 1460-byte, the throughput improvement of MP over that of SDN+HFD according to (7) is only $21.1 \%$ (as shown in Table IV). This small improvement, however, does not match the large, 200\%-plus improvement, observed in Fig. 13. On the one hand, this is a pleasant result adding to the merit of MP for large-scale Wi-Fi cellular networks. On the other hand, this observation also begs for an answer as to what is the fundamental cause for this large improvement. The analysis for the multi-cell case is an interesting subject for further investigation in future work in view of the large improvement of MP.

\section{Conclusion}

We have investigated an 802.11-like MAC protocol based on cross-layer carrier sensing, referred to as MP, aimed at solving the $\mathrm{EN}$ and $\mathrm{HN}$ problems in large-scale Wi-Fi networks. We believe this is a first paper to propose and examine the merit of MAC-address-based Physical carrier sensing, a concept that exploits cross-layer design.

Our performance study finds that MP gives surprisingly good throughput and fairness performance compared with other previously studied schemes, particularly with respect to the carrier-sensing schemes in conventional 802.11 (in this paper we have used IEEE $802.11 \mathrm{~b}$ as an example) wireless networks. The throughput achieved by MP is typically more than twice that of the $802.11 \mathrm{~b}$ basic-access mode, and more than four times that of the $802.11 \mathrm{~b}$ RTS/CTS mode, for various network-parameter settings (e.g., different settings of node density, packet size, etc.). At the same time, MP achieves Jain's Fairness Index that is nearly twice those of the $802.11 \mathrm{~b}$ basic-access and RTS/CTS modes.

Besides comparison with the conventional 802.11 schemes, we have also compared MP with other schemes previously proposed by us to deal with EN and HN, including the SDN and SDN+HFD schemes. Although there is generally a trade-off in network design between throughput and fairness so that superior performance in one generally results in inferior performance in the other, we have demonstrated that MP can achieve superior performance on both measures compared with these other schemes. This fully demonstrates the merit of using PHY-MAC cross-layer design for the implementation of carrier sensing.

Our original motivation for MP was simply the elimination of the RTS/CTS overhead. While the improvement of MP over SDN+HFD is as predicted by the overheadreduction analysis for a single-cell Wi-Fi network with only one AP, the improvement of MP over SDN+HFD in a

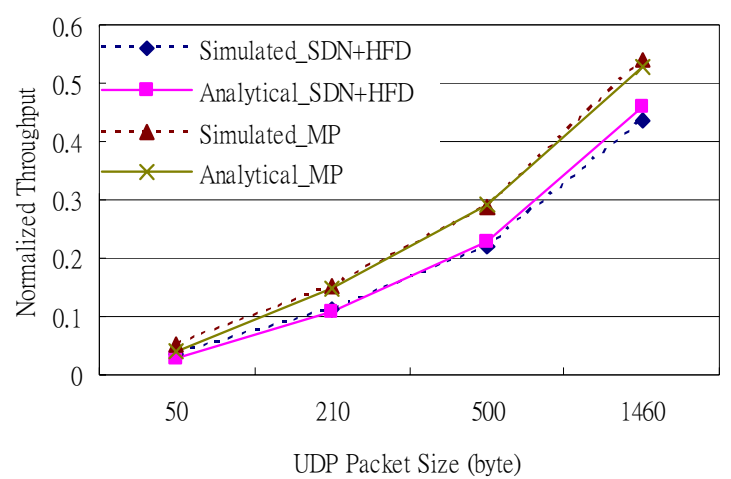

Fig. 14. Simulated and analytical normalized throughput of MP and SDN+HFD in a single-cell situation when different sizes of UDP packets are used

large-scale multi-cell Wi-Fi network with multiple adjacent APs is much larger than is indicated by the overheadreduction analysis alone. This could be due to other factors such as further robustness against EN in MP (since a major difference between the single-cell and multi-cell cases is that $\mathrm{EN}$ is absent in the former but is a major reason against throughput scalability in the latter). In view of its magnitude, the analysis of the multi-cell case to identify the root cause for the large improvement is an interesting subject for further investigation.

\section{References}

[1] P. C. Ng, S. C. Liew, L. Jiang, "Achieving Scalable Performance in Large-Scale IEEE 802.11 Wireless Networks," IEEE Wireless and Communications and Networking Conference, Mar. 2005.

[2] L. Jiang, "Improving Capacity and Fairness by Elimination of Exposed and Hidden Nodes in 802.11 Networks," M.Phil Thesis, The Chinese University of Hong Kong, Jun. 2005.

[3] M. S. Gast, 802.11 Wireless Networks: Definitive Guide, O'Reilly, Sebastopol, CA, 2002.

[4] W. Wang, S. C. Liew, V. O. K. Li, "Solutions to Performance Problems in VoIP over 802.11 Wireless LAN," IEEE Trans. on Vehicular Technology, vol. 54, no. 1, Jan. 2005.

[5] L. Jiang, S. C. Liew, "Removing Hidden Nodes in IEEE 802.11 Wireless Networks," IEEE Vehicular Technology Conference, Sep. 2005.

[6] P. Gupta, P. R. Kumar, "The Capacity of Wireless Network," IEEE Trans. on Information Theory, vol. 46, no. 2, pp.388-404, Mar. 2002.

[7] Xu, S.; Saadawi, T., "Does the IEEE 802.11 MAC protocol work well in multihop wireless ad hoc networks?," IEEE Communications Magazine, Vol. 39, No. 6, pp. 130 - 137, Jun. 2001.

[8] IEEE Std. 802.11-1999, "IEEE Standard for Wireless LAN Medium Access Control (MAC) and Physical Layer (PHY) specifications", ISO/IEC 8 802-11: 1999(E), Aug. 1999.

[9] "The Network Simulator - ns2," http://www.isi.edu/nsnam/ns/.

[10] R. Jain, D. M. Chiu and W. Hawe, "A Quantitative Measure of Fairness and Discrimination for Resource Allocation in Shared Systems," Technical Report, Digital Equipment Corporation, DEC-TR-301, 1984.

[11] "Voice Over IP - Per Call Bandwidth Consumption [Voice Quality]-Cisco Systems," http://www.cisco.com/en/US/tech/ tk652/tk698/technologies tech note09186a0080094ae2.shtml\#c onfigvoice.

[12] G. Bianchi, "Performance Analysis of the IEEE 802.11 Distributed Coordination Function", IEEE Journal on Selected Areas in Communications, vol. 18, No. 3, Mar. 2000. 